\title{
Polycystic Ovarian Morphology is Associated with Hyperandrogenemia and Insulin Resistance in Women with Polycystic Ovary Syndrome (PCOS)
}

Neoklis AG ${ }^{1 *}$, Anastasia KA ${ }^{1}$, Damianaki $K^{1}$, Nikolaos DR ${ }^{1}$, Markantes $\mathbf{G}^{1}$, Papadopoulos $\mathbf{V}^{1}$, Adonakis GL ${ }^{1}$, Decavalas $\mathbf{G}^{1}$ and Panidis $\mathrm{D}^{2}$

${ }^{1}$ Department of Obstetrics and Gynecology, Division of Reproductive Endocrinology, University of Patras Medical School, Patras, Greece

2Division of Endocrinology and Human Reproduction, Department of Obstetrics and Gynecology, Aristotle University of Thessaloniki, Thessaloniki, Greece

"Corresponding author: Neoklis AG, MD, PhD, Department of Obstetrics and Gynecology, Division of Reproductive Endocrinology, University of Patras Medical School, Patras, Greece, Tel: +302610999835; Fax: +302610993982; E-mail: neoklisg@hol.gr

Receiving date: Feb 29, 2016; Accepted date: Mar 25, 2016; Publishing date: Mar 30, 2016

Copyright: $\odot 2016$ Neoklis AG, et al. This is an open-access article distributed under the terms of the Creative Commons Attribution License, which permits unrestricted use, distribution, and reproduction in any medium, provided the original author and source are credited.

\begin{abstract}
Objectives: The aim of the present study was to evaluate the impact of polycystic ovarian morphology in the hormonal and metabolic features of the "classical" phenotypes of PCOS.

Design: The study included 1275 Caucasian women with PCOS with a mean age of $24.25 \pm 5.79$ years and a mean BMI of $26.80 \pm 7.03 \mathrm{~kg} / \mathrm{m}^{2}$. Diagnosis of PCOS was based on the 2003 Rotterdam ESHRE/ASRM-Sponsored PCOS Consensus criteria. Two phenotypes, matched for age and BMI were compared: Phenotype I (n=620) which included PCOS women with biochemical hyperandrogenemia and/or clinical hyperandrogenemia, chronic anovulation and polycystic ovarian morphology on ultrasound (PCO). Phenotype II ( $=400$ ) which included PCOS women with biochemical hyperandrogenemia and/or clinical hyperandrogenemia and chronic anovulation, without PCO. These phenotypes were further subdivided in normal weight and obese PCOS women.
\end{abstract}

Results: PCOS women of Phenotype I had higher LH/FSH ratio $(p<0.001)$, higher Testosterone $(p<0.01), \Delta 4$ Androstenedione $(p<0.001)$ and $17-\mathrm{OH}$ progesterone levels $(p<0.001)$, and higher Free Androgen Index (FAI) values $(p<0.01)$ compared to Phenotype II. With the exception of fasting glucose levels, all other indices of insulin resistance (fasting insulin, fasting glucose/insulin ratio, QUICKI and HOMA2IR) document an association between Phenotype I and greater insulin resistance in overweight/obese PCOS women.

Conclusions: In conclusion, in "classical" phenotypes of polycystic ovary syndrome (PCOS), polycystic ovarian morphology is associated with more severe hyperandrogenemia and deranged LH/FSH ratio. In overweight/obese PCOS subjects, PCO is positively correlated with insulin resistance.

Keywords: PCOS; Ovarian volume; Follicular number; Ovarian stroma; Hyperandrogenemia; Insulin resistance

\section{Introduction}

Polycystic ovary syndrome (PCOS) is the commonest endocrine disease of reproductive-aged women [1]. According to the National Institute of Health (NIH) criteria (1990), PCOS is diagnosed upon the concurrent presence of hyperandrogenism and/or hyperandrogenemia and menstrual dysfunction, with other known disorders excluded [2] These criteria define the so called "classical" phenotypes of the syndrome. A broader definition was suggested by the Rotterdam conference, sponsored by the ESHRE/ASRM in 2003, according to which, PCOS could be defined when at least two out of the following three features are present: oligo-anovulation, clinical and/or biochemical hyperandrogenemia and polycystic ovarian morphology on ultrasound [3]. There has been a long debate concerning the definition of PCOS, disputing the role of hyperandrogenemia as a cardinal feature of the syndrome. Additional milder phenotypes, characterized by the presence of either hyperandrogenemia and polycystic ovarian morphology (PCO) or anovulation and PCO have been included in PCOS, according to the revised ESHRE/ASRM criteria [4,5], still phenotypes that include biochemical hyperandrogenemia and chronic anovulation ("classic" PCOS) appear to be the most severely affected with respect to androgen levels, insulin resistance and obesity [5-7].

The cardinal argument in favor of the Rotterdam categorization was the use of polycystic ovarian appearance as surrogate for classical markers of hyperandrogenemia [8]. Although some authors [9-11] advocate that polycystic ovarian morphology has demonstrated satisfactory predictive value in diagnosing PCOS, this is not still universally accepted. It has been proposed that obesity differentially affects PCOS phenotypes. In particular, greater BMI and hip perimeter have been positively correlated with ultrasonographic PCO morphology, hirsutism and menstrual disorders in PCOS women $[12,13]$. The aim of the present study was to evaluate the impact of polycystic ovarian morphology in the hormonal and metabolic features of the "classical" phenotypes of PCOS.

\section{Materials and Methods}

\section{Subjects}

The study included 1275 Caucasian women with PCOS with a mean age of $24.25 \pm 5.79$ years and a mean BMI of $26.80 \pm 7.03 \mathrm{~kg} / \mathrm{m}^{2}$. Diagnosis of PCOS was based on the 2003 Rotterdam ESHRE/ASRMSponsored PCOS Consensus criteria [2], which include at least two of 
Citation: Neoklis AG, Anastasia KA, Damianaki K, Nikolaos DR, Markantes G, et al. (2016) Polycystic Ovarian Morphology is Associated with Hyperandrogenemia and Insulin Resistance in Women with Polycystic Ovary Syndrome (PCOS). J Steroids Horm Sci 7: 169. doi: $10.4172 / 2157-7536.1000169$

Page 2 of 5

the following: (i) oligo- and/or anovulation (ANOV), (ii) biochemical hyperandrogenemia and/or hyperandrogenism (clinical signs of high androgen levels) (HA), and (iii) polycystic ovaries on ultrasound (PCO). Biochemical hyperandrogenemia was defined as serum testosterone levels and/or serum Free Androgen Index being higher than two standard deviations above the mean levels of a normal control population. Clinical hyperandrogenism was defined as the presence of hirsutism, acne, or androgenic alopecia. Hirsutism was assessed by Ferriman-Gallwey scale (patients with scores of 8 or greater were considered hirsute) [14].

Chronic anovulation was defined as a menstrual cycle of less than 21 or more than 35 days. Ovulation was defined as a menstrual cycle of $28 \pm 2$ days, and/or blood progesterone levels $>5.0 \mathrm{ng} / \mathrm{mL}$ in two consecutive cycles. Exclusion criteria were congenital adrenal hyperplasia, androgen secreting tumors and Cushing's syndrome. All subjects had normal thyroid, kidney and liver function and none had excessive alcohol intake. No woman reported use of any medication that could interfere with normal function of the hypothalamicpituitary-gonadal axis during the last semester. The study complied with the principles of the Helsinki declaration, Institutional Review Board was obtained and all subjects provided written informed consent.

In order to evaluate the effect of PCO morphology on the hormonal and metabolic parameters of our PCOS patients with "classical" phenotypes (presenting biochemical and/or clinical hyperandrogenemia and chronic anovulation, from the whole cohort of PCOS women two specific phenotypes, matched for age and BMI were further analyzed: Phenotype I $(\mathrm{n}=620)$ which included PCOS women with biochemical hyperandrogenemia and/or clinical hyperandrogenemia, chronic anovulation and PCO. Phenotype II $(n=400)$ which included PCOS women with biochemical hyperandrogenemia and/or clinical hyperandrogenemia and chronic anovulation, without PCO.

Furthermore, in order to evaluate the association of both obesity and PCO morphology with the hormonal and metabolic parameters of the PCOS population, the studied populations of Phenotypes I and II $(\mathrm{n}=1020)$ were divided in normal weight $\left(\mathrm{BMI} \leq 25 \mathrm{Kg} / \mathrm{m}^{2}\right)$ and overweight/obese $\left(\mathrm{BMI}>25 \mathrm{Kg} / \mathrm{m}^{2}\right)$. Subsequently, we compared normal weight PCOS women of Phenotype I $(n=319)$ with normal weight PCOS women of Phenotype II $(n=201)$ and overweight/obese PCOS women of Phenotype I $(n=301)$ with overweight/obese PCOS women of Phenotype II $(n=199)$. The compared subgroups had no significant differences regarding age and BMI.

\section{Anthropometric and hormonal measurements}

Weight, height, as well as waist (W) and hip (H) circumferences were measured in all women. Body weight was measured using analogue scales and in light clothing; height was measured bare-foot using a stadiometer. Body mass index (BMI, $\mathrm{kg} / \mathrm{m}^{2}$ ) was calculated by dividing weight by height squared (kg per square metre) to assess obesity. Waist circumference (W) was obtained as the smallest circumference at the level of the umbilicus. Baseline blood samples were collected between days 3 and 7 of the menstrual cycle after a spontaneous or progestin-induced bleeding episode, following an overnight fast. At the same day, transvaginal ultrasound examination was performed (3-D, 5-9 MHz transducer, Voluson E6-General Electric) in order to evaluate polycystic ovarian morphology, namely increased ovarian volume $\left(>10 \mathrm{~cm}^{3}\right)$, increased follicular number $(12$ or more follicles measuring $2-9 \mathrm{~mm}$ in diameter) even in a single ovary or increased ovarian stroma (cut-off value: $1.95 \mathrm{~cm}^{2}$ ) and stroma/total area (S/A) ratio (cut-off value: 0.34$)[10,11]$. Mean number of follicles and mean ovarian volume were also assessed. Ovarian volume was calculated according to the formula: Ovarian Volume $=\pi / 6 \times$ (ovarian length $\times$ ovarian height $\times$ ovarian width). All ultrasounds were performed by the same experienced clinician.

The circulating levels of follicle stimulating hormone (FSH), luteinizing hormone ( $\mathrm{LH}$ ), Testosterone ( $\mathrm{T}), \Delta 4$ Androstenedione (A), dehydroepiandrosterone-sulfate (DHEAS), 17-OH progesterone (17$\mathrm{OH}-\mathrm{P})$, sex-hormone-binding globulin (SHBG), glucose (Glu) and insulin (Ins) were measured. The Free Androgen Index (FAI) was calculated as Testosterone $(\mathrm{nmol} / \mathrm{L}) \times 100 / \mathrm{SHBG}(\mathrm{nmol} / \mathrm{L})$ [15]. QUICKI index was calculated as the product of the equation $1 / \log$ (fasting insulin) $+\log$ (fasting glucose), while HOMA2IR index calculator was downloaded from http://www.OCDEM.ox.ac.uk.

All hormonal assays and plasma glucose determination were carried out at the Department of Biochemistry of the Aristotle University of Thessaloniki School of Medicine. Plasma glucose concentrations were measured using a glucose oxidase technique with an auto analyzer (Roche/Hitachi 902; Roche Diagnostics GmbH, Manheim, Germany).

LH and FSH levels were measured with an enzyme-linked immunoassay (EIA), using commercial kits (Nichols Institute Diagnostics, CA, USA). Testosterone was measured with a Direct RIA kit (Sorin, Biomedica); $\Delta 4$ Androstenedione with a Gamma Coat [125I] RIA kit (Incstar Corp.); DHEAS with direct RIA solid-phase coated tubes (Zer Science Based Industries Ltd); 17-OH progesterone with an ImmuChem Double Antibody [125I] RIA kit (ICN Pharmaceuticals, Inc.); insulin with a Coat-A-count Insulin kit (Diagnostic Products Corp.); and SHBG with an immunoradiometric assay (IRMA) kit (SHBG: [125I] IRMA Kit, Orion Diagnostica). The intra-assay coefficients of variation (CV) were $1.5 \%$ for $\mathrm{FSH}, 0.7 \%$ for $\mathrm{LH}, 3.8 \%$ for insulin, $4.1 \% 200$ for $17-\mathrm{OH}$ progesterone, $1.3 \%$ for testosterone, $5.9 \%$ for $\triangle 4$ Androstenedione, $9.4 \%$ for DHEAS and $5.8 \%$ for SHBG. The average inter-assay CV were $3.2 \%$ for FSH, $1.7 \%$ for $\mathrm{LH}, 4.4 \%$ for insulin, $6.3 \%$ for $17-\mathrm{OH}$ progesterone, $2.2 \%$ for testosterone, $9.2 \%$ for $\Delta 4$ Androstenedione, $12.1 \%$ for DHEA-S and $7.8 \%$ for SHBG.

\section{Statistics}

All statistical procedures were performed using SPSS 18.0 for Windows (IBM SPSS Statistics, IBM software). Values are expressed as mean \pm standard deviation. Test for normality was done using Kolmogorov-Smirnov test.

Distribution comparison between groups was done using the independent $\mathrm{t}$-test for variables following normal distribution and the non-parametric Mann-Whitney test for variables not following normal distribution. Bonferroni correction for multiple comparisons has been applied. Values were considered to be statistically significant at $\mathrm{p}<0.05$.

\section{Results}

\section{The effect of PCO morphology (Phenotype I vs. Phenotype II)}

\section{Hormonal features}

PCOS women of Phenotype I had higher LH levels $(\mathrm{p}<0.001)$, lower FSH levels $(\mathrm{p}<0.01)$ and higher LH/FSH ratio $(\mathrm{p}<0.001)$ (Figure1). 
Citation: Neoklis AG, Anastasia KA, Damianaki K, Nikolaos DR, Markantes G, et al. (2016) Polycystic Ovarian Morphology is Associated with Hyperandrogenemia and Insulin Resistance in Women with Polycystic Ovary Syndrome (PCOS). J Steroids Horm Sci 7: 169. doi: $10.4172 / 2157-7536.1000169$

Page 3 of 5

In addition, they had higher Testosterone $(\mathrm{p}<0.01), \quad \Delta 4$ Androstenedione $(\mathrm{p}<0.001)$ and $17-\mathrm{OH}$ progesterone levels $(\mathrm{p}<0.001)$, higher Free Androgen Index (FAI) values $(\mathrm{p}<0.01)$ and lower Testosterone/ $\Delta 4$ Androstenedione (T/A) ratio $(\mathrm{p}<0.01)$ (Figure 1). On the other hand, no significant difference was observed regarding DHEAS and SHBG levels among the two subgroups (Figure 1).

\section{Insulin resistance}

In all indices of insulin resistance studied (fasting glucose and insulin, glucose/insulin ratio, QUICKI and HOMA2IR indices), no significant differences were noted (Figure 1).

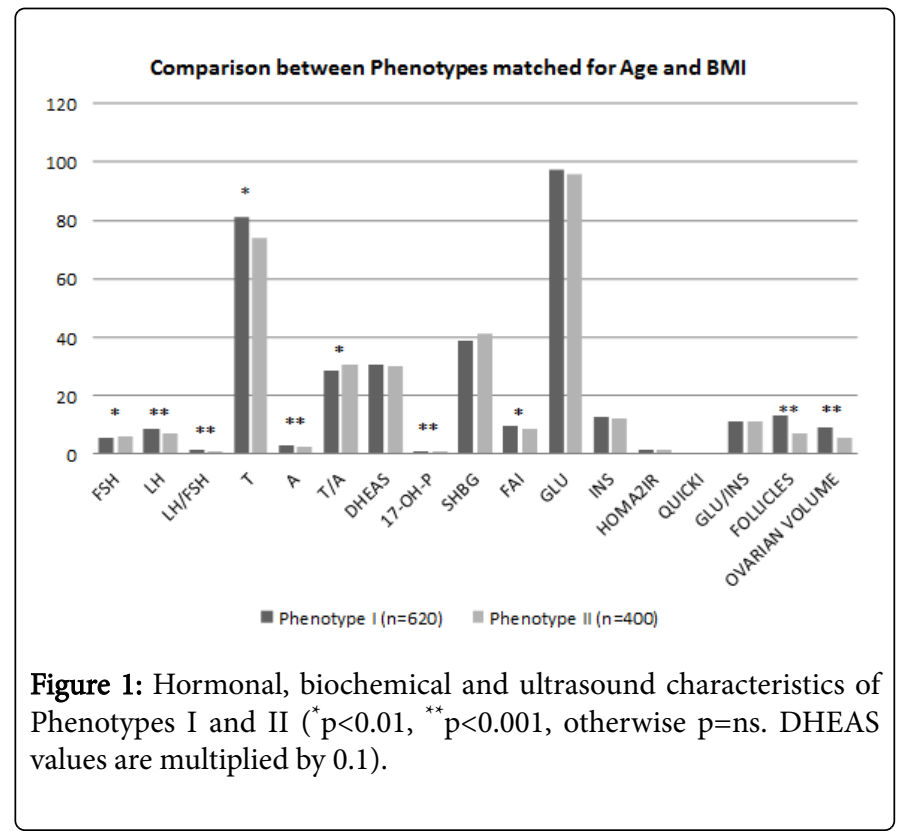

\section{The effect of PCO morphology and obesity}

\section{Normal weight women (Phenotype I vs. Phenotype II)}

\section{Hormonal features}

Normal weight PCOS women of Phenotype I had higher LH levels $(\mathrm{p}<0.001)$, lower FSH levels $(\mathrm{p}<0.05)$ and higher LH/FSH ratio $(\mathrm{p}<0.001)$ compared with normal weight PCOS patients of Phenotype II (Figure 2).

In addition, normal weight PCOS women of Phenotype I had higher Testosterone $(\mathrm{p}<0.05), \Delta 4$ Androstenedione $(\mathrm{p}<0.001), 17-\mathrm{OH}$ progesterone $(\mathrm{p}<0.001)$ and Free Androgen Index (FAI) values $(\mathrm{p}<0.01)$ and lower Testosterone/ $\Delta 4$ Androstenedione (T/A) ratio $(\mathrm{p}<0.001)$ (Figure 1), whereas no significant difference in DHEAS and SHBG levels was observed (Figure 2).

\section{Insulin resistance}

PCO morphology did not seem to affect insulin resistance in normal weight PCOS subjects, with fasting glucose and insulin, glucose/insulin ratio, QUICKI and HOMA2IR indices presenting no significant differences between the groups (Figure 2).

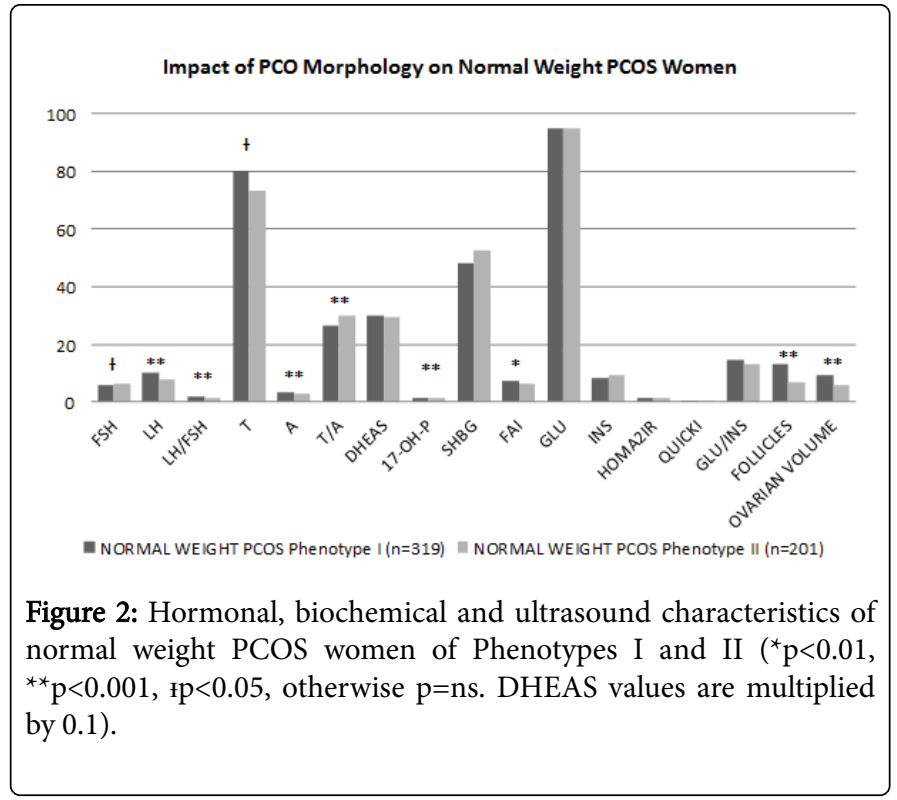

\section{Overweight/obese women (Phenotype I vs. Phenotype II)}

\section{Hormonal features}

Obese PCOS women of Phenotype I had higher LH levels $(\mathrm{p}<0.001)$ and LH/FSH ratio $(\mathrm{p}<0.001)$ and a clear tendency for lower FSH levels $(\mathrm{p}=0.062)$ compared to those of Phenotype II (Figure 3). In addition, they had higher $\Delta 4$ Androstenedione levels $(\mathrm{p}<0.001)$ and marginal differences in Testosterone $(\mathrm{p}=0.05)$ and $17-\mathrm{OH}$ progesterone levels $(\mathrm{p}=0.04)$, indicating a tendency towards elevated levels in Phenotype I (Figure 3). On the contrary, no significant difference was found concerning DHEAS and SHBG levels as well as Testosterone/ $\Delta 4$ Androstenedione (T/A) ratio (Figure 3).

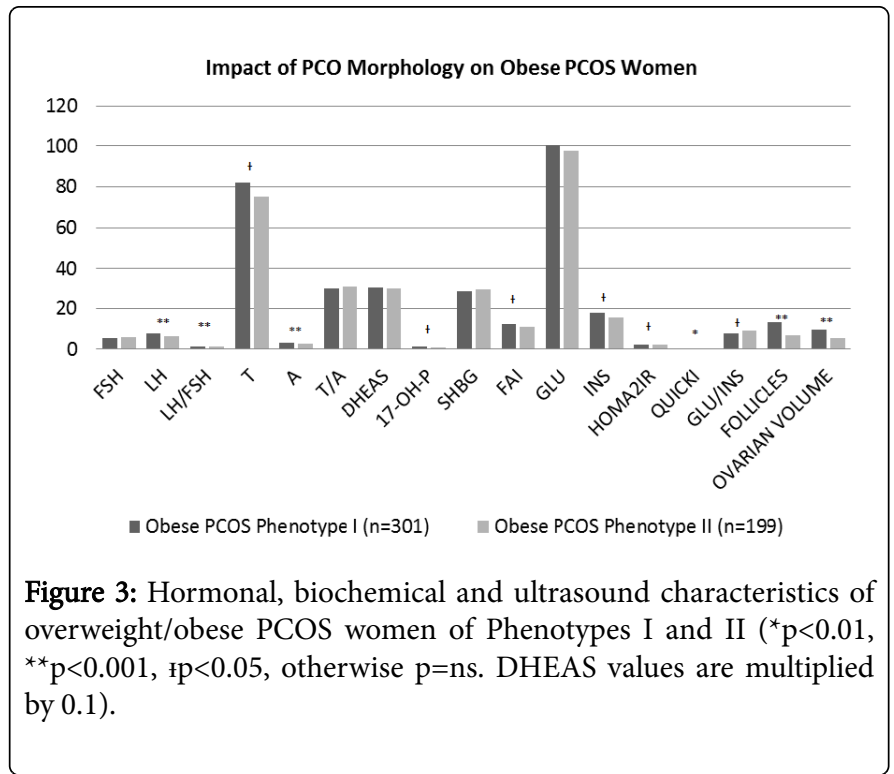

\section{Insulin resistance}

With the exception of fasting glucose levels, all indices of insulin resistance (fasting insulin, fasting glucose/insulin ratio, QUICKI and 
Citation: Neoklis AG, Anastasia KA, Damianaki K, Nikolaos DR, Markantes G, et al. (2016) Polycystic Ovarian Morphology is Associated with Hyperandrogenemia and Insulin Resistance in Women with Polycystic Ovary Syndrome (PCOS). J Steroids Horm Sci 7: 169. doi: $10.4172 / 2157-7536.1000169$

Page 4 of 5

HOMA2IR indices) document a greater impairment in insulin sensitivity, implying an association between Phenotype I and insulin resistance in overweight/obese PCOS women (Figure 3).

\section{Ovarian morphology}

Finally, as expected by definition of the study groups, women of Phenotype I had increased mean number of follicles $(p<0.001)$ and mean ovarian volume $(\mathrm{p}<0.001)$ compared to patients of Phenotype II, regardless of BMI based sub-classification (Figures 1-3).

\section{Discussion}

In the present study, polycystic ovarian morphology (PCO) on ultrasound tended to be associated with increased androgen and $\mathrm{LH}$ levels and impaired LH/FSH ratio. When dividing PCOS women into lean and overweight/obese, PCO on ultrasound tended to be associated with increased insulin resistance (IR) in overweight/obese, but not in lean PCOS women.

Strong evidence supports the relationship between ovarian follicle abnormalities and increased androgen levels. Excessive recruitment of small follicles could be attributed to intra-ovarian hyperandrogenism [16]. A positive correlation between the number of follicles of $2-5 \mathrm{~mm}$ in diameter and both testosterone and androstenedione levels has been reported [17]. On the other hand, follicular arrest could be indirectly androgen dependent given that follicles of $2-5 \mathrm{~mm}$ in diameter have been considered as the strongest independent determinant of follicular arrest. Although testosterone levels have been reported to be significant predictors of increase in stroma amount as well as follicular number and ovarian volume [18], serum testosterone levels have not been shown to be a significant independent determinant of follicular arrest [16]. The latter is contradictory with the established correlation between hyperandrogenemia and oligoanovulation [19]. However, oligomenorrhea has been reported in some patients with PCO without overt hyperandrogenism [20], while normal menstrual cycles occur in patients with PCO and hyperandrogenism [21]. Therefore, it could be speculated that serum hyperandrogenism is not a prerequisite for the existence of intra-ovarian hyperandrogenism. Hyperandrogenism exerts an indirect effect on follicular arrest via 2-5 $\mathrm{mm}$ follicle excess [16]. Consequently, follicular number could serve as surrogate for hyperandrogenism and oligoanovulation in PCOS. Biochemical indices of hyperandrogenism that characterize PCOS have been reported to be highly associated with echogenic features of PCO [7,22]. Higher androstenedione levels have been demonstrated in women with PCOS as well as healthy women with PCO as the sole finding compared to women with normal ovaries [23]. Indeed, the results of the present study confirm the above findings by depicting increased serum Testosterone, $\triangle 4$ Androstenedione and $17-\mathrm{OH}$ progesterone in PCOS women with PCO morphology compared to PCOS women without PCO.

When dividing PCOS women according to their BMI, a statistically significant strong positive relationship between PCO and indices of insulin resistance in phenotypes I and II was documented in overweight/obese PCOS women. Overweight/obese women with phenotype I had marginally higher circulating androgens than women with phenotype II and appeared to be more insulin resistant than the latter as evidenced by HOMA2IR and QUICKI indices of insulin resistance, as well as by glucose to insulin ratio. The same findings, concerning only the QUICKI index of IR have been documented in a recent study of our group including $1 / 3$ of the women enrolled in the present study [24]. In the present study, the enrolment of larger numbers of PCOS women made this association evident for HOMA2IR as well.

The association between obesity, IR and PCO morphology could be explained by the contributing effect of IR on chronic anovulation of PCOS. Insulin resistance in obese PCOS women could be attributed to obesity as well as PCOS status per se. Insulin, through its own receptor, acts synergistically to LH enhancing steroidogenesis in PCOS theca cells [25], while it is also responsible for the increase of adrenal steroids in PCOS by enhancing the sensitivity of adrenals to ACTH [26]. Actually, hyperinsulinism has been shown to exert both quantitative and qualitative negative effects on the ovulatory status in PCOS. Indices of insulin resistance have been correlated with both reduced proportion of selectable follicles (6-9 $\mathrm{mm}$ follicles) and derangement of granulosa cells [16,27]. Furthermore, androgenic effect on excessive recruitment of small follicles has been shown to be mediated by upregulation of granulosa cell insulin-like growth factor receptor [28]. On the other hand, it seems that hyperinsulinism and/or insulin resistance could not be the primary cause of anovulation of PCOS, since contradictory data dispute the establishment of a strong relationship between hyperinsulinism and anovulation. Consequently, hyperinsulinism and/or insulin resistance has been viewed as an unspecific "second hit" that further worsens follicular arrest [16]. In conditions of severe hyperandrogenism, such as "classical" phenotypes of PCOS, the relationship between insulin resistance and follicular abnormalities seems to be overshadowed by the strong impact of increased androgen levels on follicular arrest and becomes apparent only by the additive effect of obesity.

The role of ovarian ultrasound in the diagnosis of PCOS has been largely debated over the last decade. Although polycystic ovarian morphology was included in the Rotterdam criteria for the definition of the syndrome, many clinicians remain skeptical regarding its significance, as it is a common finding in healthy women (up to 25\%) and the documentation of its presence depends on the examiner. Furthermore, the strong correlation of hyperandrogenemia with adverse metabolic outcomes has led to the latter gaining the central role in PCOS diagnostic criteria, with Androgen Excess Society defining PCOS as a "predominantly hyperandrogenic syndrome" in its position statement [29]. However, various studies have proven that polycystic ovarian morphology on ultrasound (PCO) correlates well with serum and, more importantly, intra-ovarian hyperandrogenism which play a key role in follicular arrest and anovulation characterizing PCOS. The results of our study confirm the above, showing a correlation of PCO with more severe hyperandrogenemia in "classical" PCOS phenotypes and with insulin resistance in overweight/obese patients. More studies are needed in order to clarify whether ovarian ultrasound could be useful as a marker of increased risk for adverse hormonal and metabolic parameters in women with "classic" PCOS.

A possible limitation of our study is that, due to limited accuracy of the ultrasound used, we were not able to obtain meaningful measurements from our patients regarding ovarian stroma and stroma/total area to help us classify them to phenotype I or II. Hence, such results are not mentioned.

\section{References}

1. Azziz R, Woods KS, Reyna R, Key TJ, Knochenhauer ES, et al. (2004) The prevalence and features of the polycystic ovary syndrome in an unselected population. J Clin Endocrinol Metab 89: 2745-2749. 
Citation: Neoklis AG, Anastasia KA, Damianaki K, Nikolaos DR, Markantes G, et al. (2016) Polycystic Ovarian Morphology is Associated with Hyperandrogenemia and Insulin Resistance in Women with Polycystic Ovary Syndrome (PCOS). J Steroids Horm Sci 7: 169. doi: $10.4172 / 2157-7536.1000169$

Page 5 of 5

2. Zawadski JK, Dunaif A (1992) Diagnostic criteria for polycystic ovary syndrome: towards a rational approach. In: Dunaif A, Givens JR, Haseltine FP, Merriam GR (eds.) Polycystic ovary syndrome, Blackwell Scientific Publications, Boston, MA, pp: 377-384.

3. The Rotterdam ESHRE/ASRM-Sponsored PCOS Consensus Workshop Group (2004) Revised 2003 consensus on the diagnostic criteria and long term health risks related to polycystic ovary syndrome. Fertil Steril 81: 19-25.

4. Azziz R (2006) Controversy in clinical endocrinology: diagnosis of polycystic ovarian syndrome: the Rotterdam criteria are premature. J Clin Endocrinol Metab 91: 781-785.

5. Welt CK, Gudmundsson JA, Arason G, Adams J, Palsdottir H, et al. (2006) Characterizing different subsets of polycystic ovary syndrome as defined by the Rotterdam criteria: The impact of weight on phenotype and metabolic features. J Clin Endocrinol Metab 91: 4842-4848.

6. Dunaif A (1997) Insulin resistance and the polycystic ovary syndrome: mechanism and implications for pathogenesis. Endocr Rev 18: 774-800.

7. Panidis D, Tziomalos K, Macut D, Delkos D, Betsas G, et al. (2012) Crosssectional analysis of the effects of age on the hormonal, metabolic, and ultrasonographic features and the prevalence of the different phenotypes of polycystic ovary syndrome. Fertil Steril 97: 494-500.

8. Dewailly D, Pigny P, Soudan B, Catteau-Jonard S, Decanter C, et al. (2010) Reconciling the definitions of polycystic ovary syndrome: the ovarian follicle number and serum anti-Müllerian hormone concentrations aggregate with the markers of hyperandrogenism. J Clin Endocrinol Metab 95: 4399-4405.

9. Chen Y, Li L, Chen X, Zhang Q, Wang W, et al. (2008) Ovarian volume and follicle number in the diagnosis of polycystic ovary syndrome in Chinese women. Ultrasound Obstet Gynecol 32: 700-703.

10. Balen AH, Laven JS, Tan SL, Dewailly D (2003) Ultrasound assessment of the polycystic ovary: international consensus definitions. Hum Reprod Update 9: 505-514.

11. Fulghesu AM, Ciampelli M, Belosi C, Apa R, Pavone V, et al. (2001) A new ultrasound criterion for the diagnosis of polycystic ovary syndrome: the ovarian stroma/total area ratio. Fertil Steril 76: 326-331.

12. Kiddy DS, Sharp PS, White DM, Scanlon MF, Mason HD, et al. (1990) Differences in clinical and endocrine features between obese and nonobese subjects with polycystic ovary syndrome: an analysis of 263 consecutive cases. Clin Endocrinol (Oxf) 32: 213-220.

13. Tena G, Moran C, Romero R, Moran S (2011) Ovarian morphology and endocrine function in polycystic ovary syndrome. Arch Gynecol Obstet 284: 1443-1448.

14. Ferriman D, Gallwey JD (1961) Clinical assessment of body hair growth in women. J Clin Endocrinol Metab 21: 1440-1447.

15. Vermeulen A, Verdonck L, Kaufman JM (1999) A critical evaluation of simple methods for the estimation of free testosterone in serum. J Clin Endocrinol Metab 84: 3666-3672.

16. Jonard S, Dewailly D (2004) The follicular excess in polycystic ovaries, due to intra-ovarian hyperandrogenism, may be the main culprit for the follicular arrest. Hum Reprod Update 10: 107-117.
17. Dewailly D, Catteau-Jonard S, Reyss AC, Maunoury-Lefebvre C, Poncelet E, et al. (2007) The excess in 2-5 $\mathrm{mm}$ follicles seen at ovarian ultrasonography is tightly associated to the follicular arrest of the polycystic ovary syndrome. Hum Reprod 22: 1562-1566.

18. Pache TD, de Jong FH, Hop WC, Fauser BC (1993) Association between ovarian changes assessed by transvaginal sonography and clinical and endocrine signs of the polycystic ovary syndrome. Fertil Steril 59: 544-549.

19. Diamanti-Kandarakis E, Papailiou J, Palimeri S (2006) Hyperandrogenemia: pathophysiology and its role in ovulatory dysfunction in PCOS. Pediatr Endocrinol Rev 3: 198-204.

20. Dewailly D, Catteau-Jonard S, Reyss AC, Leroy M, Pigny P (2006) Oligoanovulation with polycystic ovaries but not overt hyperandrogenism. J Clin Endocrinol Metab 91: 3922-3927.

21. Carmina E, Lobo RA (2001) Polycystic ovaries in Hirsute women with normal menses. Am J Med 111: 602-606.

22. Diamanti-Kandarakis E, Panidis D (2007) Unravelling the phenotypic map of polycystic ovary syndrome (PCOS): a prospective study of 634 women with PCOS. Clin Endocrinol (Oxf) 67: 735-742.

23. Kyei-Mensah AA, LinTan S, Zaidi J, Jacobs HS (1998) Relationship of ovarian stromal volume to serum androgen concentrations in patients with polycystic ovary syndrome. Hum Reprod 13: 1437-1441.

24. Panidis D, Tziomalos K, Misichronis G, Papadakis E, Betsas G, et al. (2012) Insulin resistance and endocrine characteristics of the different phenotypes of polycystic ovary syndrome: a prospective study. Hum Reprod 27: 541-549.

25. Nestler JE, Jakubowicz DJ, de Vargas AF, Brik C, Quintero N, et al. (1998) Insulin stimulates testosterone biosynthesis by human thecal cells from women with polycystic ovary syndrome by activating its own receptor and using inositolglycan mediators as the signal transduction system. J Clin Endocrinol Metab 83: 2001-2005.

26. Moghetti P, Castello R, Negri C, Tosi F, Spiazzi GG, et al. (1996) Insulin infusion amplifies 17a-hydroxycorticosteroid intermediates response to adrenocorticotropin in hyperandrogenic women: apparent relative impairment of 17,20-lyase activity. J Clin Endocrinol Metab 81: 881-886.

27. Welt CK, Taylor AE, Fox J, Messerlian GM, Adams JM, et al. (2005) Follicular arrest in polycystic ovary syndrome is associated with deficient inhibin A and B biosynthesis. J Clin Endocrinol Metab 90: 5582-5587.

28. Vendola K, Zhou J, Wang J, Famuyiwa OA, Bievre M, et al. (1999) Androgens promote oocyte insulin-like growth factor I expression and initiation of follicle development in the primate ovary. Biol Reprod 61: 353-357.

29. Azziz R, Carmina E, Dewailly D, Diamanti-Kandarakis E, EscobarMorreale HF, et al. (2006) Criteria for defining polycystic ovary syndrome as a predominantly hyperandrogenic syndrome: an Androgen Excess Society guideline. J Clin Endocrinol Metab 91: 4237-4245. 